\title{
Oral Mucocele and its Surgical Approach as Treatment: Case Series
}

\author{
Tania Saskianti, DDS, PhD, Angela Faustina Kartono, DDS, \\ Ayudia Rifki, DDS, Yufita Fitriani, DDS and Pradita Agung Kurnia, DDS \\ Department of Pediatric Dentistry, Faculty of Dental Medicine, Universitas Airlangga, Surabaya, Indonesia
}

\begin{abstract}
A common benign lesion in children's oral cavity is mucocele on the lower lips that originates from the accumulation of mucous due to local trauma and a lip-biting habit.

Lip-biting is often motivated by a psychological condition of anxiety. Mucoceles are painless but can be bothersome for patients when eating and speaking. Mucoceles can affect the general population but are more common among the young. The etiology of oral mucoceles may vary, and surgical treatment is best chosen for its convenience, childfriendliness, and high treatment success rate.
\end{abstract}

Awareness education for children and parents is necessary to eliminate a lip-biting habit. If the pattern persists due to anxiety, it is essential to inquire more about the etiology and consult a professional psychologist.

Key Words: children; mucocele; surgical

\section{INTRODUCTION}

Oral mucocele is a common lesion of the oral mucosa that results from an alteration of minor salivary glands. Mucocele involves mucin accumulation causing limited swelling. It affects patients 11 to 17 years old and can appear at any oral mucosa site containing minor salivary glands. ${ }^{1}$ The swelling is usually caused by local trauma and obstruction of the salivary gland ducts. Based on the causes, two types of mucoceles exist - retention and extravasation. Retention mucocele appears due to a decrease or absence of glandular secretion due to the salivary gland ducts' blockage. Extravasation mucocele results from a broken salivary gland duct that spills into the soft tissues around the gland. ${ }^{2}$ Local trauma, biting habit, or both are the main etiological factors for extravasation mucocele of the lower labial mucosa. ${ }^{3}$

In children, extravasation mucoceles are more common than the retention mucoceles type. ${ }^{4}$ There is no clinical difference between retention and extravasation mucoceles. Mucoceles present as a bluish, soft, and transparent cystic swelling that frequently resolves spontaneously. The blue color is caused by vascular congestion and tissular cyanosis Paper presented in the Temu IImiah Nasional 8 (TIMNAS 8) FKG Universitas Airlangga 2020.

Corresponding author: Tania Saskianti, DDS, PhD

Department of Pediatric Dentistry

Faculty of Dental Medicine

Universitas Airlangga

Surabaya, Indonesia

Email: tania-s@fkg.unair.ac.id of the tissue above. An assessment and anamnesis should be carried out correctly. The final diagnosis is obtained from the histopathological examination..$^{5}$ Dentists should look for previous trauma; information such as the lesion's location, history of the trauma, rapid appearance, variation in size, bluish color, and consistency are crucial. Mucoceles are mobile lesions with soft and elastic consistency 
depending on how much tissue is present on the lesion. Early examination is necessary to confirm the diagnosis and prevent an exacerbation of the disease. ${ }^{2}$

In this paper, unusual findings of four mucocele cases were encountered in the past brief periods for approximately two months. This later brings up the various presumptions of their etiologies, one of which was a psychological factor. Jani et al. ${ }^{6}$ mentioned the psychological element to be correlated to lip-biting habits. The anxiety of starting a new academic year may play a role in this etiology. Anxiety in children can be discovered from several distinguished behaviors. Lower lip-biting is one often found in this state. Therefore, mucoceles are commonly found on their lower lip. Mucoceles are painless but can be bothersome for patients when they eat and speak.

Mucoceles are usually asymptomatic but sometimes can cause discomfort by interfering with speaking, chewing, or swallowing. Conventional treatment is commonly completed by excising the surrounding mucosa and glandular tissue down to the muscle layer. A simple incision of the mucocele would drain the lesion but could reappear when the wound heals. There is no need for treatment if superficial extravasation mucoceles resolve spontaneously. Small mucoceles can be removed entirely with the marginal glandular tissue before suturing. In the case of larger mucoceles, marsupialization could be done to avoid damage to vital structures. ${ }^{2}$

This case series is published with the parents' consent without exposing their identities. It aims to describe a series of mucocele cases under different clinical settings, their diagnosis, and the identification of the most appropriate therapy for an excellent clinical outcome.

\section{CASE REPORT}

\section{Case 1}

A four-year-old female child came to the Pediatric Dentistry Clinic Universitas Airlangga Dental Hospital with a chief complaint of swelling on her lower lip. This was the first time this bump appeared, which was there for approximately a month. Her mother explained that the child had a lip-biting habit since one year ago.

The swelling was diagnosed as mucocele, an extravasation type, after anamnesis and clinical examination. The bump is
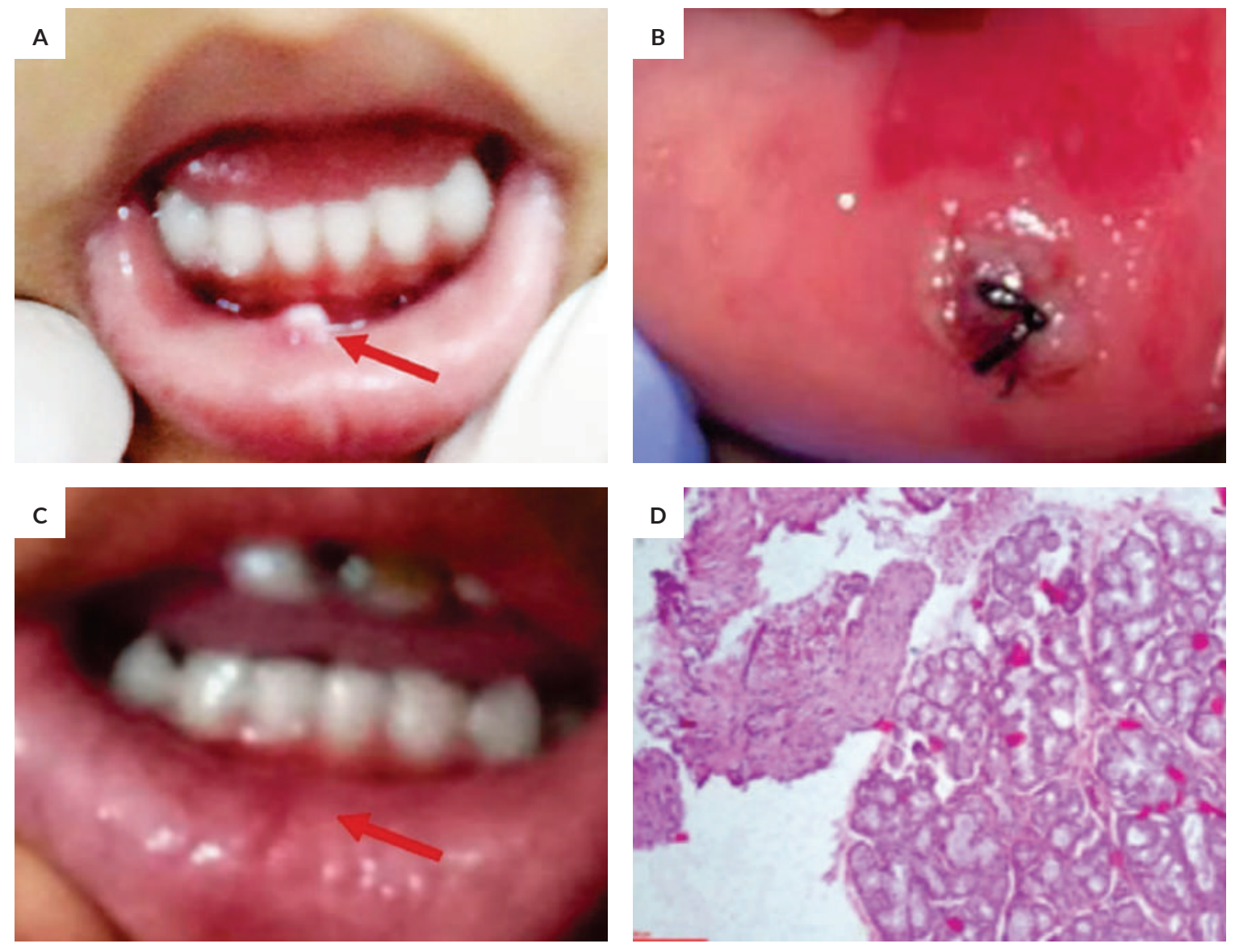

Figure 1. Mucocele Excision using Scalpel. (A) Intraoral view of mucocele on the lower lip before surgery. (B) sutures placed (C) a week post-excision mucocele. (D) Hematoxylin Eosine section under 20x magnification showing granulation tissue encapsulated by dense fibrous connective tissue. 
localized, compressible, was bluish, and has a soft fluid-filled nodule in a circular shape approximately $3.5 \mathrm{~mm}$ in diameter (Figure 1A). Considering the size and the functional disturbances due to the swelling, an excision of the lesion was performed.

Complete excision was done under local anesthesia using scalpel No. 15. The surgical site was first swabbed with $10 \%$ povidone-iodine. The excision was semilunar shape, and after the removal of the mucocele, it was sutured with 4-0 silk suture. The resected lesion was later sent for histological analysis. In the end, postoperative instructions were given, and an analgesic was prescribed. The parents and patient were instructed to eliminate the lip-biting habit. Histological findings confirmed the diagnosis as mucocele (Figure 1C). After a week, the lesion healed, no remnant was seen, and no complaint from the patient.

\section{Case 2}

A 14-year-old female child came with her parents to the Pediatric Dentistry Clinic Universitas Airlangga Dental Hospital with the chief complaint of swelling in the left lower lip region. Swelling initially emerged and gradually grew. A round, solitary, fluctuant swelling was on the inner aspect of the lower lip in 31,32 areas, $\pm 3 \mathrm{~mm}$ in diameter. The swelling was slightly bluish compared to the adjacent mucosa. The child had also reported trauma due to biting on the lower lip five months ago. The swelling was painless, and no past medical history of fever or malaise. On intraoral examination, erupting 11 and 21 were seen in crossbite relation (Figure 2A).

This case was diagnosed as an extravasation-typed mucocele based on a history of trauma and clinical features. The primary etiology was crossbite malocclusion from biting the lip. Conventional surgical treatment was planned and was explained to the parents. Once the parent's concern was taken, treatment was performed.

An incision was first made to split the overlying mucosa, and the mucocele was resected from the base. The specimen was sent for histopathological examination to confirm the diagnosis (Figure 2C). The incision was sutured and removed after seven days. Orthodontic treatment is planned to correct the malocclusion.
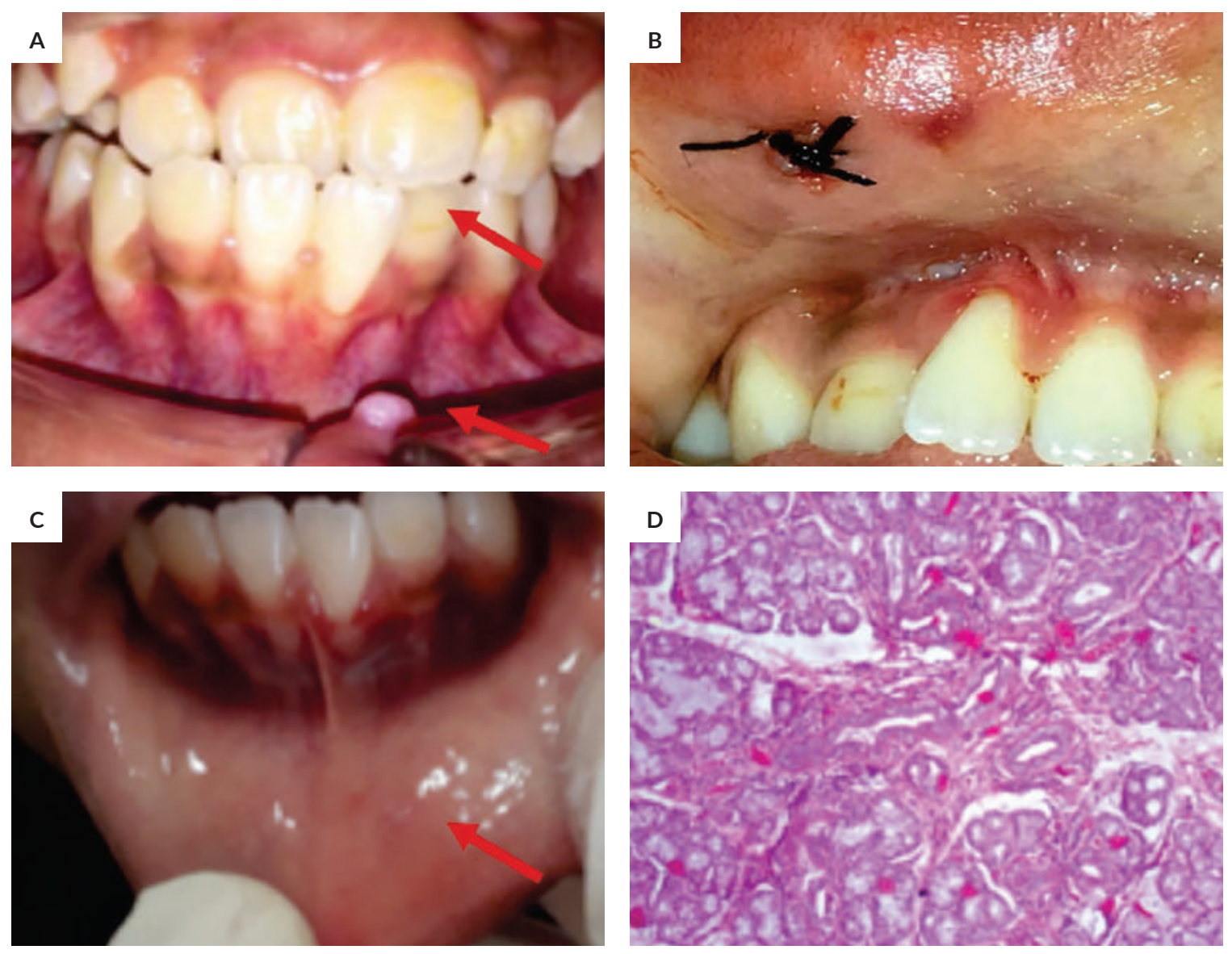

Figure 2. Pre and seven days post mucocele surgical excision on the lower lip. (A) Mucocele on the lower lip and 11, 12 in crossbite relation, 21 edge to edge relation. (B) sutures placed (C) postoperative condition. (D) Histological findings confirmed mucocele as the area of spilled mucin surrounded by connective tissue. 


\section{Case 3}

An 11-year-old male child came with his parent to the Pediatric Dentistry Clinic Universitas Airlangga Dental Hospital with the chief complaint of swelling in the right lower lip region. The swelling was in the lower lip's inner aspect between 83, 84 regions (Figure 3A) for the past six months. It was initially small and progressed to be larger to the present stage. The child has a lip-biting habit. On intra oral examination, the lesion was soft, fluctuant, and palpable, with no temperature increase. It was oval and painless. The upper right canines have partially erupted with a detailed cusp.

The case was diagnosed as an extravasation mucocele; the histological findings also confirmed the diagnosis (Figure 3C). Parents were given a detailed treatment plan after taking the parent's concerns. The incision was made using Scalpel No. 15. In the end, two sutures were removed seven days later (Figure 3B). The patient was instructed to stop biting his lips.
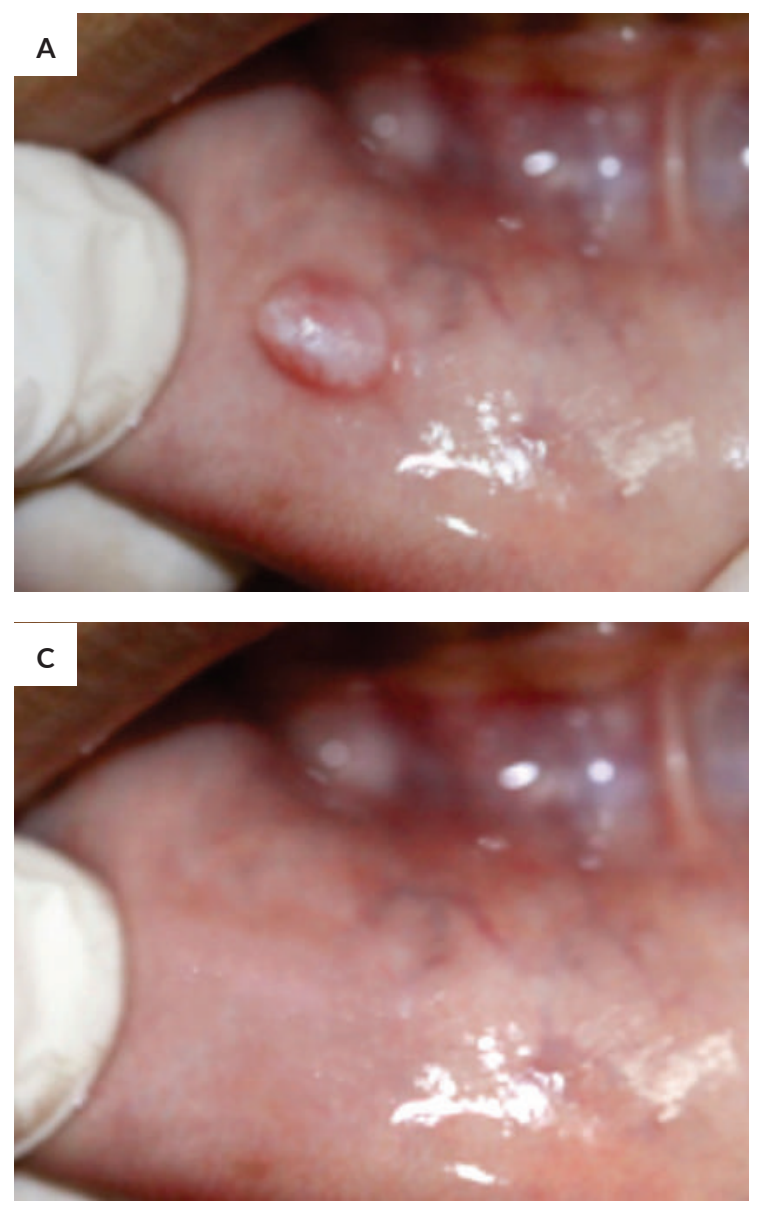

\section{Case 4}

A nine-year-old female patient reported to the Pediatric Dentistry Clinic Universitas Airlangga Dental Hospital with a chief complaint of swelling in the left lower lip region. The presenting illness showed that swelling was present in the lower lip's inner aspect in 31, 32 regions for the past three months. The bump was small and painless. The patient may have a habit of biting his left lower lip; he has no fever or malaise.

Clinical examination revealed a well-defined, raised solitary, pale pink, oval, translucent nodular growth with a smooth surface; the diameter was approximately $1 \mathrm{~cm}$ in size (Figure 4A). It was compressible, non-tender to touch, and has a sessile base. Based on history and clinical examination, a provisional diagnosis of mucocele was established.

Surgical removal of the lesion was planned and performed under anesthesia by placing an incision vertically, splitting the overlying mucosa, and resecting the mucocele from the base. A histopathological examination was made to support the diagnosis. The wound was sutured and removed seven days later (Figure 4B).
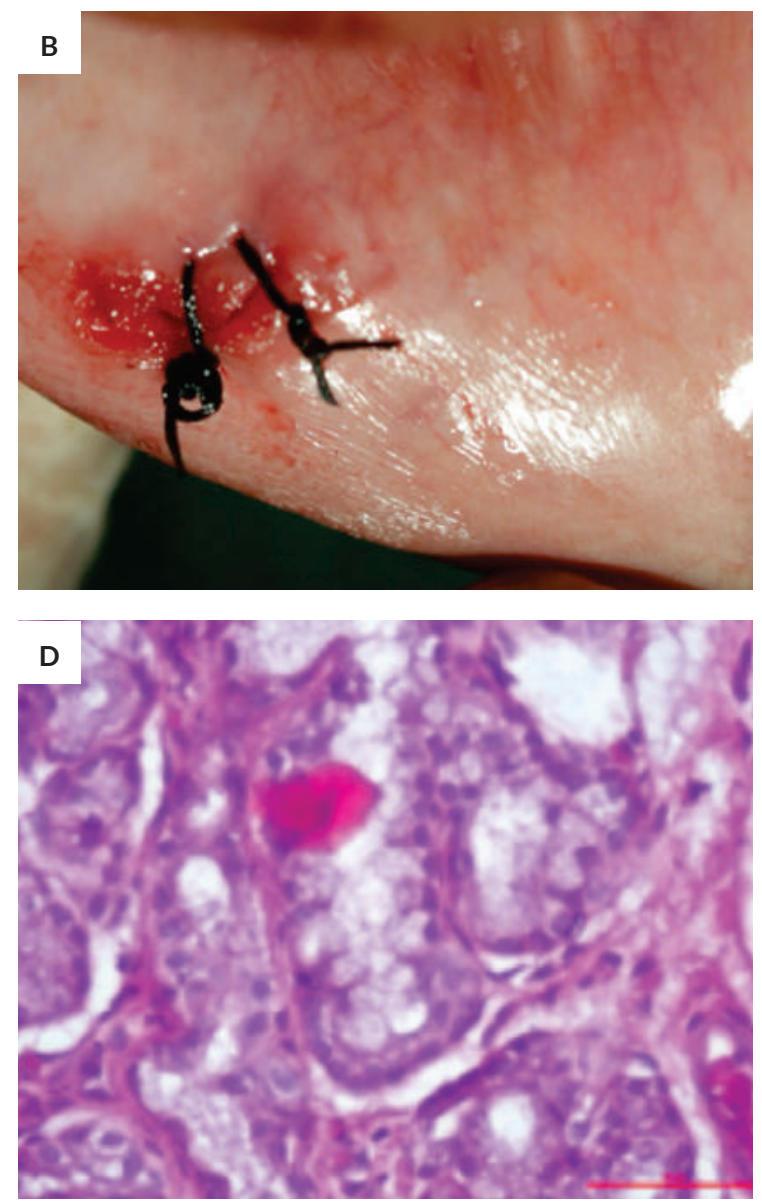

Figure 3. Mucocele Excision using a scalpel on the lower lip. (A) Intraoral view of mucocele on the lower lip before surgery. (B) sutures placed. (C) Post-excision mucocele. (D) Resected mucocele tissue under the Hematoxylin-eosin coloring. 

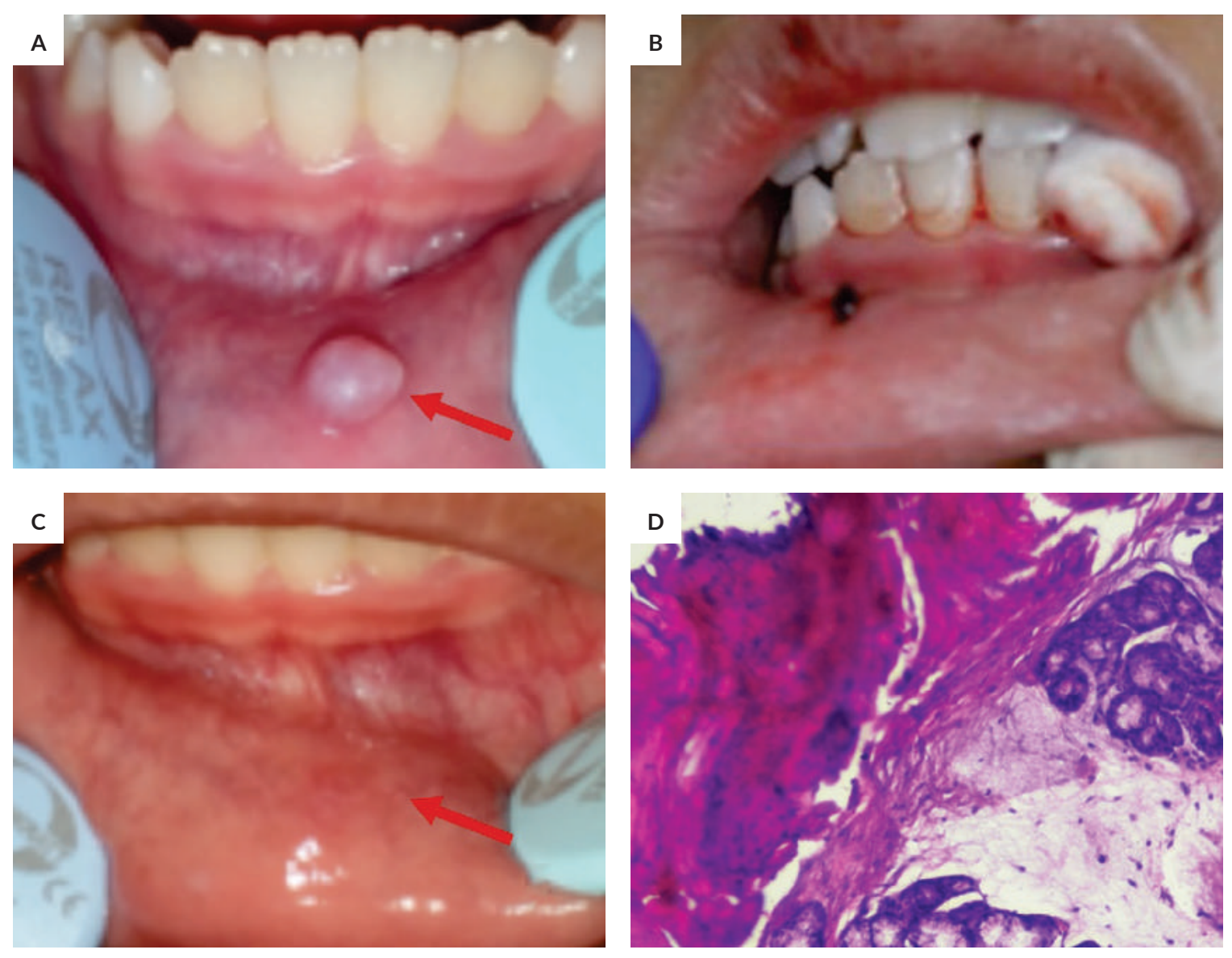

Figure 4. Mucocele Excision using a scalpel on the lower lip. (A) Intraoral view of mucocele on the lower lip before surgery. (B) Sutures placed. (C) Seven days post-excision. (D) Histological appearance of resected mucocele tissue.

\section{DISCUSSION}

In these cases, the occurrences are encountered on a similar range period of the new academic year for schools. Thus, the lip-biting habit may occur due to a demanding environment that children have to face when they were expected to understand their responsibility, control their childish behavior, and follow some new rules. These may cause children to feel frustrated and depressed, leading to lip-biting. ${ }^{6}$

Oral mucocele is one of the most common benign lesions. Although oral mucocele is harmless, it can form a permanent bump on the oral surface if left untreated, affecting overall aesthetics and oral function such as chewing and speaking.

The incidence of mucocele lesions in the general population is $0.4-0.8 \%$, with exiguous or almost no difference between males and females. ${ }^{2,7}$ In children's pathology, there were 735 cases of oral mucoceles over 30 years.

Meanwhile, $\mathrm{Wu}$ et al. reported that around 11.6-21.8\% of pediatric oral biopsies were mucoceles. ${ }^{8}$ Daniels and Mohamed stated that teenagers and children were commonly affected. ${ }^{9}$ Additionally, Yamasoba et al., Oliveira et al., and More et al. studies they mentioned that $65 \%$ of their patients with oral mucoceles were under 20 years of age. ${ }^{10}$ In the cases discussed above, the three cases were females and one male. All were children below 18 years old.

Oral mucocele is commonly found on the lower lip. In Japan and Brazil, mucocele cases of the lower lip accounted for $77.9 \%$ and $83.3 \%$, respectively. ${ }^{8}$ Less common sites for the occurrence are buccal mucosa, anterior lateral tongue, and floor mouth.

In this case series, the lesion sites were predominantly located in similar locations on one side of the lower lip. This was closely related to its most common etiology, trauma, possibly caused by the teeth' exertion or dental braces on the lower lip. ${ }^{11}$

Continuous physical trauma can cause a leakage of salivary secretion into the surrounding submucosal tissue, typically on the lower lip, thus creating an extravasation-type mucocele. ${ }^{5}$ On the third case, the mucocele was presumably aggravated by the upper right canines that partially erupted, making a continuous trauma in that area. This intensified the progress of the oral mucocele. 
According to Soo-Hyung, 25.7\% common contributory factor for an oral mucocele to occur is due to lip-biting. ${ }^{12}$ All of the remaining cases above were caused by lip-biting habits that were presumed to be related to psychological stress. Jani et al. mentioned in their study the correlation of psychological stress to lip-biting. ${ }^{7}$ The chance of pressures that children are starting a new academic year may cause this harmful habit.

In the cases described, the occurrences were encountered in a similar period - new academic school year. Thus, the lip-biting habit may be due to a demanding environment that children have to face, such as the expectation to understand their responsibility, control childish behavior, and follow new rules. These may cause children to feel frustrated and depressed, leading to lip-biting habits. ${ }^{6}$

Oral mucoceles are described as fluid-filled vesicles or blisters in superficial mucosa or as a fluctuant nodule deep within the connective tissue. ${ }^{7,13}$ These bluish, soft, transparent vesicles can rupture spontaneously and leave ulceration on the surface that heals within few days. Their deep blue color results from tissular cyanosis and vascular congestion of the tissue above and accumulation of mucin beneath. The color may vary depending on the lesion's dimension, the vicinity of the mucosal surface, and the overlying tissue's resiliency. ${ }^{2}$

Apart from its distinguishable appearance, histopathological examinations were also taken to determine the diagnosis. Histologically, two patterns were seen; they were called the retention and the extravasation type. Extravasation type begins with an intact epithelium-lined duct dilated to form a cyst, it then is filled with mucin and inflammatory debris (retention cyst type) or extravasated mucin within the stroma, often associated with granulation tissue, a brisk inflammatory response, and foamy histiocytes, without epithelium (extravasation type). ${ }^{14}$

In all cases, there were obstructive changes in the minor salivary glands, and epithelioid macrophages can be seen among the mucin. There were inflammatory reactions such as macrophages containing phagocytized mucus in all three cases supporting their subjective and objective examinations. Thus, as a result of this, we concluded they were all extravasated types. ${ }^{10}$

By far, there are several methods to treat oral mucoceles. They are the conventional approach using a scalpel or electrosurgery, and nonsurgical method using corticosteroid injections, cryosurgery, and electrocautery. ${ }^{15}$

Corticosteroid injection is a single intralesional steroid injection, preceded by aspiration of the cyst. It causes the pseudocyst wall to collapse and triggers a severe inflammatory reaction of the wall resulting in marked fibrosis. ${ }^{15}$

Cryosurgery is a method of lesion destruction by rapid freezing. The lesion is frozen, and the resulting necrotic tissue is allowed to slough off spontaneously. The advantage of this technique includes no intraoperative or postoperative bleeding, with minimal surgical defects and scarring. The disadvantage of this technique requires trained skills because it is difficult to gauge the freezing depth. If it is too deep, it can cause damage to deeper structures. ${ }^{15}$

The electrocautery technique to remove mucoceles has a minimal bleeding advantage. The electrode cuts both on its side and tip. The hemostasis is immediate and consistent, the wound is painless, and the tip is self-disinfecting. Disadvantages of electrocautery are an anesthetic agent for cutting requirement, unavoidable burning flesh odor, and low tactile sense. ${ }^{15}$

According to Baurmash, a conventional method by surgical approach is the most common choice. There are three possible surgical approaches in the management of oral mucoceles: 1 . Simple excision of the lesion; 2. Marsupialization; 3. Complete excision of the lesion along with the associated salivary gland. ${ }^{16}$

In this case series, we opted for conventional surgical excision with a scalpel. This method was preferred due to its low recurrence of relapse despite its necessity to be careful in its procedure. ${ }^{17}$ Conventional surgical excision ensures that both affected and adjacent glands are eliminated along with the pathologic tissue before the wound starts to heal to minimize the risk of recurrence. This treatment is manageable for children, inexpensive, and is performed under local anesthesia. ${ }^{18}$ The comparative study conducted by Bahadure et al. on the success of conventional surgical management of oral mucocele in a group of subjects in the transitional period from mixed dentition to permanent dentition proved that conventional surgical management of mucoceles in pediatric patients is the definitive treatment modality. On this consideration, we prefer to use the conventional surgical technique.

After complete excision, suturing should also be carefully performed to promote healing and prevent the adjacent glands or ducts' rupture. Postoperative instruction to eliminate the lip-biting habit should be given afterward. In addition, awareness education for children and parents is necessary to stop the lip-biting habit. If the practice persists due to a child's anxiety, it is essential to inquire more about the etiology of the addiction and consult a professional psychologist.

After a week post-operation, patients felt more comfortable because there was no interfering bump. They reported that chewing and speech function has improved. The intensity and frequency of biting the lower lip have also gradually decreased.

\section{CONCLUSION}

Oral mucocele is a relatively common benign lesion in children. Traumatism is one of the frequent causes of its occurrence. Various clinical parameters must be taken into consideration in opting for the proper treatment to prevent a recurrence. Awareness education for children and parents is also essential to eliminate the harmful lip biting habit. Professional psychologists can opt for a different approach. 


\section{Statement of Authorship}

All authors participated in the data collection and analysis and approved the final version submitted.

\section{Author Disclosure}

All authors declared no conflicts of interest.

\section{Funding Source}

This paper was funded by the Community Service Grant from Faculty of Dental Medicine Universitas Airlangga 2019.

\section{REFERENCES}

1. Mnguez-Martinez I, Bonet-Coloma C, Ata-Ali-Mahmud J, Carrillo-Garca C, Pearrocha-Diago M, Pearrocha-Diago M. Clinical characteristics, treatment, and evolution of 89 mucoceles in children. J Oral Maxillofac Surg [Internet]. 2010 [cited 2020 Oct 16];68(10):2468-71. Available from: http://dx.doi.org/10.1016/j. joms.2009.12.038

2. Ata-Ali J, Carrillo C, Bonet C, Balaguer J, Peñarrocha Diago M, Peñarrocha M. Oral mucocele: Review of the literature. J Clin Exp Dent. 2010;2(1):18-21.

3. Poulopoulos A. Salivary Mucoceles in Children and Adolescents: A Clinicopathological Study. Glob J Med Clin Case Reports. 2017;4:011-4.

4. Chaitanya P, Praveen D, Reddy M. Mucocele on lower lip: A case series. Indian Dermatol Online J [Internet]. 2017 [cited 2020 Oct 14]; 8(3):205. Available from:/pmc/articles/PMC5447343/?report=abstract

5. Nallasivam KU, Sudha BR. Oral mucocele: Review of literature and a case report. J Pharm Bioallied Sci [Internet]. 2015 Aug 1 [cited 2020 Oct 16];7(6):S731-3. Available from: /pmc/articles/ PMC4606697/?report=abstract

6. Gartika M. The effect of oral habits in the oral cavity of children and its treatment. Padjadjaran J Dent. 2008;20(2):123-9.
7. Indiarti IS, Ariawan D. A case report of mucocele. Ijcpd. 2013;9(4): 253-6.

8. Wu CW, Kao YH, Chen CM, Hsu HJ, Chen CM, Huang IY. Mucoceles of the oral cavity in pediatric patients. Kaohsiung J Med Sci [Internet]. 2011 [cited 2020 Oct 16];27(7):276-9. Available from: http://dx.doi.org/10.1016/j.kjms.2010.09.006

9. Daniels JS. Mucocele of anterior lingual salivary glands of blandin \& nuhn: A report of 5 cases | Request PDF. Saudi Dent Journals [Internet]. 2005 [cited 2020 Oct 16];17(3):154-61. Available from: https://www.researchgate.net/publication/303315162_Mucocele_ of_anterior_lingual_salivary_glands_of_blandin_nuhn_A_report_ of_5_cases

10. More CB, Bhavsar K, Varma S, Tailor M. Oral mucocele: A clinical and histopathological study. J Oral Maxillofac Pathol. 2014;18(5):72-6.

11. Jose SC, Korath Abraham K, Khosla E. Blandin and Nuhn mucocele in a pediatric patient. J Indian Soc Pedod Prev Dent. 2018;36(3): $315-8$.

12. Soo-Hyung Han, MD Sang-Oon Baek, MD Sung-No Jung M. Mucocele in the Buccal Vestibule Granular Cell Ameloblastoma of the Anterior Mandible. 2012;23(6):2012.

13. Jeffrey A. Dean and Kevin J. Donly. CHAPTER-11 Restorative Dentistry. McDonald and Averys Dentistry for the Child and Adolescent. 2016. 185-205 p.

14. Thompson LDR. Mucocele: Retention and extravasation types. Ear, Nose Throat J. 2013;92(3):106-7.

15. Gautam Y, Srivastava M. Mucocele in paediatric patients: A case series with review. Int J Appl Dent Sci. 2018;4(2):100-3.

16. Baurmash HD. Mucoceles and ranulas. J Oral Maxillofac Surg. 2003;61(3):369-78.

17. Bahadure RN, Fulzele P, Thosar N, Badole G, Baliga S. Conventional surgical treatment of oral mucocele: A series of 23 cases. Eur J Paediatr Dent. 2012;13(2):143-6.

18. Valério RA, de Queiroz AM, Romualdo PC, Brentegani LG, de PaulaSilva FWG. Mucocele and fibroma: Treatment and clinical features for differential diagnosis. Braz Dent J. 2013;24(5):537-41.

Have you read the current trends in Medical and Health Research in the Philippines?

\title{
Acta Medica Philippina The National Health Science Journal
}

\author{
Access Online: www.actamedicaphilippina.upm.edu.ph
}

